\title{
La défense belge dans le futur
}

André Dumoulin $\mid$ Attaché à l'Institut royal supérieur de défense (IRSD), professeur à l'Université de Liège. N'engage pas les institutions de référence.

$\mathrm{L}$ 'année 2015 sera une année importante pour l'avenir des forces armées belges face à un défi majeur : la rédaction d'un plan dit stratégique à dix ans dont les lignes de forces générales seront présentées par le nouveau ministre de la Défense, Stephen Vandeput (NVA, parti nationaliste flamand) fin avril. Cette ébauche de la future configuration de la défense en 2030 devra être adoptée par le Gouvernement, avant que ne soit soumis au Conseil des ministres un plan stratégique détaillé, cela avant la fête nationale du 21 juillet 2015.

Un plan directeur sera enfin soumis dans le courant de septembre. Les contraintes sont énormes, à la fois budgétaires, politiques et communautaires (cf. A. Dumoulin). À ce calendrier s'ajoutent le dossier très délicat du remplacement des avions de combat $F-16$ et plusieurs contraintes structurelles.

\section{Invariances belges}

Primo, depuis 1981 et par la suite, avec le plan Charlier et les autres plans, le pays a vécu bien des amaigrissements dont les restructurations successives des forces armées avaient davantage pour objet de répondre aux contraintes budgétaires que d'être la résultante d'une réflexion de fond assumée.

Secundo, il n'y a pas en Belgique, de la part des citoyens, et ce à la fois pour des motifs historiques, sociologiques, politiques et culturels, de véritable culture militaire partagée et encore moins de culture stratégique. La posture belge, quelque peu indifférente, n'aide en rien le soutien à l'institution ni sa représentativité au sein des instances parlementaires.

Tertio, la complexité sociétale belge, à la fois communautaire, linguistique et socio-historique, y compris l'organisation des gouvernements de coalition, rend délicates l'organisation des processus de décision dans le domaine de la défense, pourtant à assumer sur le long terme ; d'autant que chaque gouvernement futur peut détricoter les décisions prises.

\section{Méthodologie}

La préparation du plan 2015 a été organisée à la fois par le cabinet politique du ministère de la Défense, une petite équipe d'officiers supérieurs gravitant 
autour du chef de la Défense (CHOD), le général Van Caelenberge (composante Air), l'organisation d'un colloque fermé en présence d'experts et des médias le 25 février $2015^{(1)}$ et une campagne de sondages du personnel de la Défense, parallèlement à l'organisation d'auditions d'experts civils et militaires nationaux et internationaux par la commission défense de la Chambre des représentants (Parlement belge) entre janvier et février 2015 dirigée par une député NVA.

Il s'agit pour le ministre d'acquérir une série d'informations plurisectorielles avant de prendre attitude, en collaboration avec l'état-major et le gouvernement de coalition à forte dominance néerlandophone, seul le parti libéral francophone est dans la coalition exécutive.

Cependant, nous subodorons que l'élaboration d'un plan stratégique belge est, dès le départ, conditionné, inscrit et formaté par les contraintes budgétaires ; les limites du portefeuille étant posées au préalable, l'habillage l'étant aussi à partir des chiffres et non par mise en questionnements d'une vision politico-militaire à construire.

Reste que la Belgique a été le moteur de la construction européenne, fortement fédéraliste dans ses postures diplomatiques et aiguillon pour avancer dans certaines matières délicates. Il ne peut donc y avoir d'orientations stratégiques sans un lien très affirmé et très visible entre notre Royaume et la politique européenne de sécurité et de défense (PESD) et celle d'ordre transatlantique.

N'est-il pas pertinent de poser la question de savoir si, comme pour le groupe des Quatre en 2003, nous pensions notre défense nationale en des termes encore plus intégratifs ? Et si la Belgique osait accepter perdre quelques pans de sa souveraineté dans le cadre européen en matière de sécurité-défense ? Au vu de la situation internationale, quel meilleur message que celui-là : l'Union fait la force (2) et l'intégration le moyen d'y parvenir réellement.

\section{Des tâches essentielles au capacitaire}

L'impossibilité d'établir une étanchéité entre les interventions extérieures (exemples : Mali, Syrie, Irak) et le terrorisme intérieur ( $c f$. A. Dumoulin, les épisodes de Charlie Hebdo à Paris et de Verviers en Belgique en janvier 2015) et l'obligation de ne pas se refermer sur ses propres frontières au nom d'une prise de risque refusée ou du refus de solidarité entre alliés vont imposer de repenser la sécurité intérieure de la Belgique davantage en termes multinationaux (coopération).

(1) Les documents préparatoires furent placés par la suite sur le site Internet du ministre belge de la Défense.

(2) C'est aussi la devise de la Belgique ! 
Face aux hypothèses générales posées à l'horizon 2030 et aux réalités budgétaires ${ }^{(3)}$ et géographiques (taille du pays) ${ }^{(4)}$, l'armée belge devrait avoir essentiellement pour tâches de soutenir, par un choix de niches d'excellence et de compétences, les missions et opérations multinationales réalisées dans le cadre de l'UE, de l'Otan ou de l'ONU. Pourquoi des niches ? Parce qu'au final, l'armée belge hérite de plus de trente ans d'amaigrissements et que les niches sont la solution ultime à défendre, sauf à souhaiter la fin de l'institution.

Il s'agit d'anticiper les besoins et de localiser la rareté que la Belgique pourrait offrir sur étagère, en symbiose bien entendu avec les choix politiques et en aval les capacités budgétaires du pays. Penser coalition et organiser en grande partie l'armée belge grâce à cette dimension en acceptant même de perdre quelques pans de souveraineté ; l'espace belge étant fortement imbriqué par les États voisins et dans les espaces vitaux allemands, français et britanniques. Peut-être est-ce pour cela déjà que les moyens alloués pour la défense belge sont aussi limités.

Relevons que le 4 mars, la Belgique et les Pays-Bas ont signé un accord visant à instaurer d'ici à la mi-2017 une surveillance aérienne conjointe $(Q R A)^{(5)}$ de leurs deux pays, y compris le Luxembourg.

Les tâches essentielles de l'Armée belge devront se concentrer dans la proche périphérie de l'Europe, l'espace euro-méditerranéen et africain, en évitant une politique de panachage, ce saupoudrage qui a, certes, son intérêt diplomatique par visibilité plurielle du drapeau, mais qui occasionne une dispersion trop importante des moyens, une augmentation des coûts et une vulnérabilité potentielle.

Une des premières tâches générales imposées par la menace terroriste et numérique qui ne vont pas s'éteindre sera l'augmentation des moyens alloués au renseignement.

La deuxième sera, à l'avenir, de continuer à soutenir la politique pluridimensionnelle de l'UE en matière de sécurité et celle de l'Alliance.

La troisième tâche générale devra relever de l'aide à la Nation sur toutes ses formes. Au vu de la faiblesse des citoyens en matière de culture stratégique et en partie en matière de résilience, l'engagement des forces armées belges en aide à la population sera un élément important pouvant favoriser les soutiens à l'institution militaire et aider à la compréhension des spécificités et des missions de l'armée.

(3) Le budget de la défense a diminué de plus d'un quart depuis 1998. En termes réels, le budget de la défense a été réduit de 2,2 milliards de 1981 à 2014. Les dépenses de défense (modèle Otan) sont passées de $1,03 \%$ en 2013 à $0,66 \%$ en 2014 et devraient atteindre $0,5 \%$ du PIB en 2015. Les coupes estimées cumulées sur la durée de la législature du gouvernement Michel seront de près de 1,6 milliard d'euros, dont 225 millions en 2015. Cf. aussi le rapport de la Cour des comptes sous le manque de moyens alloués à la Justice et à la Défense cette année.

(4) Relevons néanmoins que la Belgique est le $9^{\mathrm{e}}$ (sur 28 de l'UE) en termes de produit intérieur brut et $8^{\mathrm{e}}$ selon le critère PIB par habitant. La dette publique est estimée à $107 \%$ du PIB.

(5) Quick Reaction Alert. 
À terme et en fonction de l'environnement géopolitique prévisible, les tâches essentielles ${ }^{(6)}$ devraient idéalement reposer sur plusieurs capacités :

- Associées à la sécurité sociétale intérieure : protection des citoyens et des infrastructures critiques ${ }^{(7)}$, appui à la police fédérale ; appui à la protection civile, récupération de ressortissants.

- De niches qui seront associées aux missions et opérations Otan et UE et qui relèveraient d'un avantage comparatif. Il pourrait s'agir de conserver-maintenir ou de disposer d'une capacité de transport aérien, d'une capacité aérienne multirôle, d'une capacité de forces spéciales, d'une capacité C3 (commandement, contrôle et communications) modulaire et projetable, d'une capacité légère paracommandos, d'une capacité médiane type Battlegroup ${ }^{(8)}$ apte aussi à répondre à une infanterie légère irrégulière, d'une capacité en renseignement électronique ${ }^{(9)}$, d'une capacité de déminage terrestre, d'une capacité maritime anti-mines, d'une capacité frégate multifonctionnelle $(\mathrm{NH}-90)$, d'une capacité de surveillance des côtes.

- À être interopérable en matière de ravitaillement en vol, logistique, munitions guidées, $C-4 \ldots$

- De formation d'unités militaires étrangères afin de responsabiliser les États fragilisés et d'engager les autorités politiques locales et lesdites armées dans des réformes conforment aux grands principes qui fondent la démocratie et la séparation des pouvoirs.

\section{Des propositions}

La Belgique devrait, dès à présent...

- Poursuivre, et c'est un truisme, une réflexion politico-militaro-industrielle sur la définition des niches qui sera proposée dans le plan stratégique 2015-2030 attendu ce printemps, parallèlement à une loi-programme (équipements) sur dix ans. Cependant, la définition des niches ne pourra être uniquement associée virtuellement à un moindre risque en laissant à nos partenaires les risques accrus par le choix des systèmes d'armes et doctrines associées. Dès lors, le choix des systèmes d'armes et des unités préservées, y compris leur formation, doivent refléter cette solidarité, ce Risk sharing. Il s'agira aussi de réfléchir sur une révision des conclusions de la Commission Rwanda (1997) qui doit être en grande partie actualisée au vu de l'environnement international, des opérations et missions belges en Afrique subsaharienne et des principes généraux sur la question des risques.

\footnotetext{
(6) Cette hypothèse pourrait amener la disparition de l'artillerie tractée de campagne, des drones Hunter (déjà programmé pour 2017) et la fermeture de l'hôpital militaire et d'autres infrastructures petites ou moyennes.

(7) Protection des infrastructures : énergie, ports d'Anvers et Liège, transport, industrie chimique et nucléaire, communications.

(8) Apte au combat, à la stabilisation, à la reconstruction, avec des moyens de combat + génie, logistique, transmission, hôpital de campagne rôle 1 .

(9) Apport aux équipages AWACS Otan et maintien d'une participation à un système satellitaire d'observation. Acquisition de mini et micro-drones (sécurité intérieure et mission extérieure), ISTAR.
} 
- Sanctuariser le budget de la défense en lien direct avec le plan stratégique, afin de créer les conditions garantissant l'opérationnalité des forces armées, la crédibilité des engagements militaires belges, la stabilité du personnel tout en permettant d'engager un plan pluriannuel des investissements dégagés des variables d'ajustement, respectant la trajectoire financière entérinée.

- Redéfinir la structure du renseignement belge au vu de l'évolution de l'environnement international et national.

- Dépasser les rivalités d'influence des composantes, contre-productive pour l'armée dans sa globalité, mais aussi éviter toute éviction d'une arme suite à l'acquisition de systèmes d'armes trop coûteux, tout en garantissant l'équilibre régional dans la répartition et la fonction des implantations dans un pays politiquement très compliqué.

- Maintenir un équilibre entre la coopération bénéluxienne, française et allemande avec leur propre spécificité. La priorité pourrait être de penser les niches belges avec " un accrochage " de la composante Terre à la Bundeswehr, de la composante aérienne à l'Armée de l'air française et de la composante Mer aux Pays-Bas, sans que cela n'empêche d'autres configurations multinationales élargies.

- Penser la question de la formation obligatoire des jeunes au civisme, sans relancer le service militaire qui est seulement suspendu en Belgique.

- Renforcer la réserve et l'entraîner prioritairement à l'aide multiforme à la nation en synergie avec la protection civile dans une nouvelle vision interdépartementale.

- Maintenir et intensifier les missions de formation des armées étrangères en général et africaines en particulier ${ }^{(10)}$ dans des coopérations assumées bilatérales (équipe de liaison et de tutorat opérationnel) ${ }^{(11)}$.

- Étudier le ratio " para-commandos » et «forces spéciales ».

- Continuer à être proactif dans la participation aux deux forces de réaction rapide que sont les Battlegroups 1500 (participation 2018) et la force de réaction rapide Otan $(N R F)$ (engagement 2016).

- Réaliser une étude indépendante sur les conséquences juridiques, budgétaires, politiques et sécuritaires du recours à certaines externalisations ; mais aussi examiner les implications autour de possibles "locations " de matériels militaires majeurs venant d'autres pays alliés ( $c f$. A. de Neve).

(10) Actuellement existe le programme de partenariat militaire (PPM) au Bénin, en République démocratique du Congo et au Burundi.

(11) Operational Mentor and Liaison Team (OMLT). 
- Être attentif à la crédibilité de la composante Terre (autour d'une brigade dite "médiane ") mise à mal et fragilisée par les orientations politiques et par le poids important dans le processus de décision des aviateurs : selon certains analystes, sa puissance de feu pourrait être inférieure à celle de l'Estonie et s'orienter vers des missions statiques de force protection, de formation et de déminage.

- Tenter d'éviter que l'acquisition de nouveaux avions de combat (F-35, Rafale, Eurofighter, Gripen) ne crée les conditions d'un déséquilibre majeur entre les forces, fragilise l'équilibre communautaire et linguistique à propos des deux bases aériennes majeures (Kleine Brogel, Florennes : 87 pilotes pour 54 F-16) qui les accueilleront, réduise fortement les retombées économiques et pose question dans le partage et le contrôle des technologies.

\section{Le mythe de Sisyphe}

Reste que le ministre de la Défense, Stephen Vandeput (NVA) a indiqué qu'aucun dossier d'investissement majeur ne sera lancé durant cette législature! Les difficultés budgétaires et les enjeux sous-jacents expliquent la raison du report du calendrier de la présentation précise du plan stratégique cette année.

Pour conclure, sachant que le curseur géopolitique est tout sauf fixe, l'impératif budgétaire est en porte à faux avec les besoins sécuritaires ${ }^{(12)}$ et avec les ambitions, certes mesurées, des autorités politiques. La sortie par le bas serait de continuer à dégraisser, l'armée étant vue comme la vache à lait, en attentant la " peau de chagrin " si ce n'est déjà fait, avec aussi les dilemmes autour de la pyramide des âges déséquilibrée et la perte de compétences au vu de la multiplication des départs à la retraite dans les toutes prochaines années. Déjà, l’année 2014 a vu certains achats postposés, une réduction dans le budget de fonctionnement et l'infrastructure.

Entre une armée qui fond sous le "soleil " (32000 à 20000 militaires en fin de législature ?) ${ }^{(13)}$, une armée belge imbriquée totalement en multinational, en soutien, ayant déjà renoncé par attrition mécanique à la conduite au sol d'opérations de haute intensité, une armée "police ", ou une armée résiduelle devant rester crédible, la marge de manœuvre reste et restera étroite ; la calculette nous revenant par la fenêtre, sachant que le présent gouvernement veut atteindre l'équilibre budgétaire en 2018 .

\section{ÉLÉMENTS DE BIBLIOGRAPHIE}

André Dumoulin : "Belgique : une défense jaune-bleu ? ", carte blanche, Europe Diplomatie \& défense, n 721, Agence Europe, Bruxelles, 31 juillet 2014.

André Dumoulin : "Belgique et terrorisme : “C’est arrivé près de chez nous” ", Tribune 603, RDN, janvier 2015.

Alain de Neve : "Special Purpose Vehicle (SPV). Quels atouts ? Quels enjeux ? Quelles incidences sur l'opérationnalité des armées? ", e-Note 16, IRSD, Bruxelles, 17 février 2015.

(12) La Cour des comptes a souligné que pour la Justice et la Défense, le budget 2015 était déjà insuffisant.

(13) Relevons que la pyramide des âges des militaires est déséquilibrée avec $46 \%$ nés dans les sixties. 